\section{Estudio de asociación de base familiar entre polimorfismos de MTHFR y mielomeningocele en Chile}

\author{
ROSA PARDO ${ }^{1,2,3}$, JOSÉ SUAZO ${ }^{4}$, SILVIA CASTILLO ${ }^{1,5}$, \\ MARCELA VARGAS ${ }^{\mathrm{a}}$, ANDREA ZALAVARI ${ }^{\mathrm{a}}$, \\ JOSÉ LUIS SANTOS ${ }^{6}$, RAFAEL BLANCO ${ }^{7}, \mathrm{KARIN} \mathrm{ROTTER}^{8}$, \\ MARGARITA SOLAR ${ }^{8}$, EVA TAPIA ${ }^{9}$
}

\section{Methylenetetrahydrofolate reductase polymorphisms as risk factors for myelomeningocele}

Background: Mandatory fortification with folic acid (FA) was implemented in Chile in 2000. Thereafter, the rate of spina bifida decreased by 52 to 55\%. Genetic abnormalities in folate metabolism may be involved in the etiology of spina bifida. Aim: To evaluate the association between myelomeningocele (MM) and c.A1298C and c.C677T polymorphisms within the coding gene for 5,10-methylenetetrahydrofolate reductase (MTHFR) in the Chilean population. Material and Methods: These polymorphisms were genotyped in 105 patients showing isolated MM, born after the onset of FA fortification, and in their parents. The transmission disequilibrium test (TDT) was performed to evaluate alterations in the transmission of both alleles and haplotypes MTHFR polymorphism. We also evaluated the presence of parent-origin-effect (POE) of alleles using the Clayton's extension of the TDT. Results: TDT analysis showed no significant distortions in the transmission of alleles or haplotypes. Moreover, although the POE showed increased risk for maternally derived allele, this risk was not statistically significant. Conclusions: The studied variants in the MTHFR gene (c.C677T and c.A1298C) do not constitute risk factors for MM in this sample of Chilean patients and their parents.

(Rev Med Chile 2014; 142: 587-592)

Key words: Myelomeningocele, folic acid, MTHRF protein, human; Spinal dysraphism.
'Sección de Genética, Hospital Clínico Universidad de Chile

2Unidad de Neonatología, Hospital

Clínico Universidad de Chile.

${ }^{3}$ Unidad de Genética, Hospital Dr. Sótero del Río.

${ }^{4}$ Instituto de Investigación en Ciencias Odontológicas, Facultad de Odontología, Universidad de Chile. ${ }^{5}$ Unidad de Citogénetica, Laboratorio, Clínica Alemana de Santiago, Santiago, Chile.

${ }^{6}$ Departamento de Nutrición, Diabetes y Metabolismo, Escuela de Medicina, Pontificia Universidad Católica de Chile. ${ }^{7}$ Programa de Genética Humana, Instituto de Ciencias Biomédicas, Universidad de Chile.

${ }^{8}$ Sociedad Pro Ayuda del Niño Lisiado (TELETON-Chile).

${ }^{9}$ Centro de Rehabilitación Infantil del Ejército (CRIE).

aEstudiante de Tecnología Médica, Escuela de Tecnología Médica, Universidad del Desarrollo.

Financiamiento: Proyecto OAIC 479/11, financiado por el Hospital Clínico Universidad de Chile, Institución que no tuvo ingerencia en los procesos de diseño de estudio, análisis e interpretación de resultados, en la preparación del manuscrito, ni en su aprobación.

Recibido el 9 de agosto de 2013, aceptado el 17 de mayo de 2014 .

Correspondencia a:

Rosa Pardo

Sección Genética, Hospital Clínico Universidad de Chile

Santos Dumont 999, 8380456,

Independencia.

Santiago, Chile.

Fono-fax: 29788513

rpardo@hcuch.cl
U na de las malformaciones congénitas más comunes son los defectos de cierre del tubo neural (DTN) los cuales afectan entre 0,5-2 casos/1.000 gestaciones en el mundo ${ }^{1}$. En Chile, su prevalencia es de 0,5-0,8/1.000 nacimientos, de los cuales cerca del $50 \%$ corresponden a espina bífida (EB). Dentro de los pacientes con $\mathrm{EB}$, la mayoría de ellos corresponden a mielomeningocele $(\mathrm{MM})^{2,3}$. Para la EB se ha postulado una etiología multifactorial, en la cual interactúan tanto factores genéticos como ambientales ${ }^{1}$. En consecuencia, varios genes han sido postulados como candidatos a ser responsables de la etiología de la $\mathrm{EB}$, los cuales están principalmente relacionados con vías metabólicas tales como la del transporte y metabolismo de la vitamina B12, los folatos y los procesos de metilación ${ }^{4}$. En este contexto, en 2000 se implementó en Chile la fortificación obligatoria 
de la harina con ácido fólico (FAF). Después de esta intervención de salud pública las tasas de EB se redujeron entre 52 y $55 \%{ }^{2,3}$.

Considerando que los niveles altos de homocisteína se asocian con un riesgo mayor de $\mathrm{DTN}^{5}$, uno de los factores de riesgo más ampliamente estudiados para estas malformaciones es el gen que codifica para la 5,10-metilen-tetrahidrofolato reductasa (MTHFR) la cual convierte el folato dietario en su forma activa, permitiendo la transformación de la homocisteína a metionina a través de su remetilación ${ }^{6}$. Las variantes no sinónimas del gen MTHFR: p.Ala222Val (c.C677T) y p.Glu429Ala (c.A1298C), que se asocian a disminución de la actividad de la enzima, han sido las más estudiadas como posibles factores etiológicos de $\mathrm{DTN}^{7}$. No obstante, se han descrito resultados controversiales respecto a la asociación de estas variantes con el riesgo de presentar DTN en varias poblaciones, inconsistencias que podrían ser explicadas por el diferente origen étnico de dichas muestras ${ }^{8-10}$. Se ha descrito además que el uso de acido fólico induciría una selección genética en favor de los alelos asociados a una menor actividad de MTHFR $(677 \mathrm{~T} \text { y } 1298 \mathrm{C})^{11,12}$.

Se ha comunicado sólo un estudio previo, publicado en 2003, enfocado en el rol de variantes de la MTHFR en la EB en Chile. En dicho estudio, Nitsche et al. ${ }^{13}$ no detectaron diferencias significativas entre la frecuencia del polimorfismo C677T entre 58 madres de pacientes con EB y 184 mujeres controles. Sin embargo, dicho estudio no especifica si estos casos pertenecen al periodo pre o post FAF. Así, el objetivo de nuestro estudio fue revaluar la asociación entre MM y la variante c.C677T de MTHFR en la población chilena en la era post-FAF, adicionando el análisis de la variante c.A1298C.

\section{Material y Métodos}

Nuestra muestra fue recolectada entre 2010 y 2012 y estuvo constituida por 105 casos de niños con MM aislado nacidos en el periodo post FAF y sus progenitores ( 86 tríos caso-progenitores y 19 dúos caso-madre). Respecto a los casos, 53,3\% eran de sexo femenino, el rango de edades fue 2 meses a 10 años y presentaban conforme al nivel anatómico de la EB la siguiente clasificación: 8 altas (cérvico-torácicas), 2 tóraco-lumbares y 95 bajas (lumbo-sacras). Los casos provienen de todos los estratos socioeconómicos (ABC1 7,6\%, C2 20\%, C3 $16,2 \%$, D $32,4 \%$ y E $23,8 \%$ ) y son atendidos en los Institutos de la Sociedad Pro Ayuda del Niño Lisiado (Teletón-Chile) de las ciudades de Santiago y Valparaíso, y en el Centro de Rehabilitación Infantil del Ejército de Chile (CRIE), en Santiago. Los padres de los casos son en su mayoría chilenos $(90,5 \%)$, encontrándose en los restantes nacionalidad peruana en 5 casos $(4,8 \%)$, brasileña en 3 casos $(2,9 \%)$, ecuatoriana en 1 caso $(1.8 \%)$ y panameña en 1 caso $(1,8 \%)$. Además los padres se reconocen como originarios de etnias chilenas en el 11,4\% de los casos (11 mapuches y 1 aymará).

El protocolo de este estudio fue aprobado por el Comité de Ética del Hospital Clínico Universidad de Chile y todos los participantes o sus padres/ tutores, dieron su consentimiento informado para participar en el.

De leucocitos de sangre periférica se extrajo el ADN genómico, empleando el método descrito por Chomczynski y Sacchi ${ }^{14}$. Las variantes c.C677T y c.A1298C del gen MTHFR fueron genotipificadas con ensayos de discriminación alélica usando sondas Taqman (Applied Biosystems, ID C_1202883_20y C_850486_20, respectivamente). Las frecuencias alélicas para estos marcadores fueron estimadas utilizando tanto los genotipos parentales como de los casos, en donde la presencia del equilibrio de Hardy-Weinberg se evaluó a través de la prueba de $\chi^{2}$ usando el paquete estadístico STATA 12.

Para evaluar el patrón diferencial de la transmisión de los alelos y los haplotipos se empleó el test de desequilibrio de transmisión (TDT) ${ }^{15}$. Dado que nuestra muestra estaba compuesta por tríos y dúos, optamos por efectuar el TDT empleando el programa UNPHASED 3.1.6, el cual aplica un algoritmo que ha demostrado ser robusto en la estimación del genotipo parental no disponible en los dúos ${ }^{16}$. Se evaluó además el efecto de origen parental (EOP) de los alelos de estas variantes, empleando la extensión de Clayton del $\mathrm{TDT}^{17}$, incorporada en el paquete STATA 12. Se incluyó este análisis adicional, dado que el EOP debiera ser considerado como un modelo de herencia en enfermedades complejas, debido a que, en algunos casos, la capacidad funcional de un alelo no es equivalente si es heredado derivado de la madre o del padre ${ }^{18}$. El EOP puede ser evaluado estratificando las transmisión de los alelos, en el TDT, para madres y padres ${ }^{19}$, lo cual puede reflejar a) la 
Polimorfismos de MTHFR y espina bífida en Chile - R. Pardo et al

Tabla 1. Frecuencias genotípicas y alélicas variantes polimórficas de MTHFR

\begin{tabular}{|c|c|c|c|c|c|c|c|c|}
\hline \multirow[t]{2}{*}{ Variante $^{a}$} & \multirow{2}{*}{$\begin{array}{l}\text { Cambio } \\
\text { codificado }\end{array}$} & \multirow{2}{*}{$\begin{array}{l}\text { Cambio } \\
\text { Proteico }\end{array}$} & \multicolumn{3}{|c|}{ Frecuencias genotípicas } & \multicolumn{3}{|c|}{ Frecuencias alélicas } \\
\hline & & & Genotipos & Progenitores & Casos & Alelos & Progenitores & Casos \\
\hline \multirow[t]{3}{*}{ rs1801133 } & c.C677T & p.Ala222Val & $\mathrm{CC}$ & 0,560 & 0,566 & C & 0,752 & 0,751 \\
\hline & & & $\mathrm{CT}$ & 0,383 & 0,372 & & & \\
\hline & & & $\mathrm{TT}$ & 0,057 & 0,062 & $\mathrm{~T}$ & 0,248 & 0,249 \\
\hline \multirow[t]{3}{*}{ rs1801131 } & c.A1298C & p.Glu429Ala & $A A$ & 0,638 & 0,604 & A & 0,790 & 0,793 \\
\hline & & & $A C$ & 0,305 & 0,378 & & & \\
\hline & & & $\mathrm{CC}$ & 0,057 & 0,018 & C & 0,210 & 0,207 \\
\hline
\end{tabular}

aDe acuerdo a dbSNP (http://www.ncbi.nlm.nih.gov/projects/SNP/).

impronta genómica como una vía importante de variación en los rasgos complejos ${ }^{20} \mathrm{ob}$ ) los efectos del genotipo materno en el ambiente uterino ${ }^{21}$, siendo este último el mecanismo referido para la actividad reducida de la MTHFR materna en los casos de DTN ${ }^{22}$.

\section{Resultados}

Las frecuencias alélicas para las variantes c.C677T y c.A1298C de MTHFR, estimada sobre los genotipos de casos y progenitores, se muestran en la Tabla 1. Dichas frecuencias se distribuyen de acuerdo al equilibrio de Hardy-Weinberg en ambos grupos (datos no mostrados). Los resultados del análisis de TDT no evidencian ninguna distorsión en la transmisión, de progenitores a progenie afectada, ni de alelos, ni de los haplotipos de estas variantes. Este resultado se observó tanto para el análisis de todos los casos (Tabla 2), así como para los casos correspondientes a MM bajos (90,4\% de los casos) (datos no mostrados). Por otra parte, aunque el análisis de EOP para ambas variantes, mostró un aumento en el riesgo de MM para los alelos derivados de la madre, estos valores no fueron significativos ni al analizar la muestra en forma global (Tabla 3 ) ni al considerar solamente los MM bajos (datos no mostrados).

Tabla 2. Resultados del TDT para alelos y haplotipos de las variantes polimórficas de MTHFR

\begin{tabular}{|ccc|}
\hline Variante & O/E $^{\mathbf{a}}$ & p-value \\
rs1801133 (c.C677T) & $56 / 58$ & 0,817 \\
rs1801131 (c.A1298C) & $46 / 45$ & 0,975 \\
Haplotipos $^{\text {b }}$ & $\mathbf{O}^{\mathbf{c}}$ & p-value \\
C-A & $138 / 132$ & 0,585 \\
C-C & $31 / 35$ & 0,157 \\
T-A & $40 / 47$ & 0,209 \\
T-C & $15 / 10$ & 0,534 \\
\hline
\end{tabular}

${ }^{a} \mathrm{O} / \mathrm{E}$ : recuento de transmisión observada/ esperada para el alelo de menor frecuencia de progenitores heterocigotos a progenie afectada. ${ }^{\text {b}}$ Orden de las variantes en los haplotipos: rs1801133- rs1801131. 'O/E: recuento de transmisión observada/ esperada para los haplotipos de progenitores heterocigotos a progenie afectada.

Tabla 3. Riesgos maternos y paternos para los alelos de las variantes polimórficas de MTHFR

\begin{tabular}{|c|c|c|c|c|}
\hline Variante & Alelo $^{a}$ & $\begin{array}{c}\text { Riesgo de herencia } \\
\text { Materna del alelo }(95 \% \mathrm{Cl})^{\mathrm{b}}\end{array}$ & $\begin{array}{c}\text { Riesgo de herencia } \\
\text { Paterna del alelo }(95 \% \mathrm{CI})^{\mathbf{b}}\end{array}$ & $\begin{array}{c}\text { p-value } \\
\text { (Razón de verosimilitud) }\end{array}$ \\
\hline c. C677T & $\mathrm{T}$ & $1,30(0,57-2,97)$ & $0,77(0,34-1,76)$ & 0,530 \\
\hline c.A1298C & C & $1,38(0,68-2,82)$ & $0,72(0,35-1,47)$ & 0,370 \\
\hline
\end{tabular}

${ }^{a}$ Alelo de menor frecuencia. El alelo de mayor frecuencia es considerado como referencia (riesgo = 1) por la extensión de Clayton del TDT. ${ }^{b}$ Intervalo de confianza estimado de acuerdo a Bland y Altman ${ }^{23}$. ${ }^{c} p$-value de la prueba de razón de verosimilitud. 


\section{Discusión}

Los resultados controversiales sobre el rol de las variantes c.C677T y c.A1298C del gen MTHFR en relación al riesgo de DTN, han llevado a algunos autores a efectuar el análisis conjunto de datos de diferentes estudios. Así, los meta-análisis resultantes de estos estudios tienen el fin de detectar asociaciones en muestras considerablemente más grandes en vez de hacerlo en reportes individuales. Dentro de estos estudios para la variante c.C677T, considerando trabajos que incluyeron principalmente poblaciones de origen caucásica, Van der Put et al. ${ }^{8}$ demostraron que dicho polimorfismo constituía un marcador de aumento de riesgo de manifestar DTN en todas las poblaciones analizadas, excepto para la italiana. Diez años después, Amorim et al. ${ }^{9}$ publicaron un meta-análisis considerando poblaciones de todos los orígenes étnicos. Estos autores, al igual que Van der Put el $\mathrm{al}^{8}$, confirmaron que la variante c.C677T era un factor de riesgo. Sin embargo, cuando estos autores estratificaron su muestra por orígenes europeolatino y europeo- no latino, los datos se mantuvieron significativos sólo para el segundo grupo. En el caso de la variante c.A1298C, un meta-análisis en población caucásica no detectó asociación entre este marcador y el riesgo de $\mathrm{DTN}^{10}$. Así, los resultados de estos meta-análisis concuerdan con nuestros hallazgos que no evidencian asociación entre variantes de MTFHR y MM.

Los resultados del presente estudio muestran la misma tendencia en relación a un reporte previo de Nitsche et al., en Chile ${ }^{13}$, en el cual no se detectaron diferencias en las frecuencias alélicas de la variante c.C677T, entre madres de niños con EB y madres de niños sanos. En base a las conclusiones extraídas de los meta-análisis antes mencionados, la ausencia de resultados significativos en las muestras chilenas podría explicarse por el origen étnico de la nuestra población contemporánea. Esta población presenta componentes tanto europeo-latinos (conquistadores españoles) como amerindios, en una mezcla iniciada en los siglos XVI y XVII con un efecto menor de migraciones caucásicas más tardías (siglo XIX $)^{24,25}$. Sin embargo, el estudio de Nitsche et al. ${ }^{13}$ muestra, para las madres de afectados, una frecuencia del alelo 677T mucho mayor que la registrada en el presente estudio $(0,457$ y 0,265 , respectivamente). El estudio de Nitsche et al. ${ }^{13}$ fue publicado el año
2003 sin especificar el período en que se reclutó las muestra analizada, por lo que no se puede descartar que se hayan incluido madres cuyo embarazo y parto ocurrió previo a la fortificación con ácido fólico. Por ello, las menores frecuencias alélicas de nuestro estudio (muestra reclutada durante 2010-2012), parecen paradójicas. Se ha postulado que el uso de acido fólico produciría una selección genética en favor de alelos asociados a una menor actividad de MTHFR (677T y 1298C) $)^{11,12}$. Por esta razón debiéramos esperar un aumento de la frecuencia de estos alelos con el paso del tiempo en la población general, resultado opuesto a lo observado en el presente estudio. Estas diferencias pueden posiblemente ser explicadas por una combinación de factores tales como las diferencias en la composición genética de las poblaciones en comparación y los efectos de las técnicas de genotipificación. Al analizar las poblaciones estudiadas en el caso de Nitsche et $\mathrm{al}^{13}$, se encuentra que incluye sólo pacientes de estrato socioeconómico bajo, en tanto que nuestra población abarca a todos los estratos, variable relevante en cuanto a composición genética en Chile $e^{25}$; por otra parte, si bien se describen en el presente trabajo más datos referentes a ancestros del caso, no es posible contrastarlos con el estudio citado, dado que no se cuenta con una acabada descripción del origen étnico en el mismo. Adicionalmente la muestra de Nitsche et al..$^{13}$ se reclutó sólo desde un centro en Santiago, mientras que la nuestra consideró dos centros en esta ciudad y uno en la ciudad de Valparaíso, ciudades que presentan alguna diferencia en el grado de mezcla amerindia de sus poblaciones ${ }^{26}$, lo que podría aumentar la diversidad de nuestra muestra. Además, el estudio de Nitsche et al. ${ }^{13}$ determinó los genotipos en base a la reacción en cadena de la polimerasa asociada al análisis de fragmentos de restricción (PCR-RFLP), mientras que nuestro estudio ensayos de PCR en tiempo real utilizando sondas fluorescentes alelo-especificas. En este contexto, usando la secuenciación directa como método de referencia de genotipificación, se ha demostrado que la técnica de PCR-RFLP presenta un porcentaje de error de genotipificación que es mayor que las técnicas basadas en PCR en tiempo reall ${ }^{27,28}$.

Dentro de los DTN, tras la FAF de la harina de trigo, las tasas de EB se redujeron entre 52-55\% en comparación con descensos de $41-50 \%$ para la anencefalia ${ }^{2,3,29}$. Si bien los autores de estos reportes 
no explicitan que el descenso de las tasas de EB sean significativamente mayores que las de anencefalia, se observa una tendencia que hace pensar que el defecto espina bífida sería más sensible a la disponibilidad de folatos ${ }^{2}$ y posiblemente los factores que originan los nuevos casos tras la FAF no tengan relación con el metabolismo de los folatos. Es así como, por ejemplo, en la misma muestra analizada en el presente estudio, hemos reportado una asociación entre variantes de genes relacionados con la homeostasis energética (SLC2A1, HK1 y $L E P R$ ) y $\mathrm{MM}^{30}$. A pesar que nuestros resultados no encontraron asociación entre variantes del gen MTHFR y la EB, consideramos que la vía metabólica del folato no puede aún ser excluida del todo como una causa de MM después de la FAF en Chile. Existen variables en otros genes involucrados en esta vía, tales como TYMS, MTHFD1, SHMT1 y MFTC que han sido involucrados en la etiología de la EB en otras poblaciones ${ }^{31,32}$. Por lo tanto, se requiere de nuevos estudios con el fin de aclarar la contribución global de esta vía metabólica en nuestro país, idealmente con tamaños muestrales mayores que permitan detectar riesgos genotípicos menores.

En un estudio previo, Volcik et al. ${ }^{7}$ analizaron la relación entre la variante c.C677T del gen MTHFR y MM (en una muestra hispánica de residentes de EEUU descendientes de mejicanos) considerando la posición del MM. Estos autores encontraron que el genotipo TT de la posición c.677 es un factor de riesgo, pero solo cuando es portadora la madre y sólo para los casos de EB alta. Este efecto del genotipo materno no fue detectado en nuestro estudio, posiblemente debido al alto número de casos de EB baja. En este contexto, los diferentes sitios de cierre del tubo neural podrían ser controlados por la expresión de diferentes genes ${ }^{33}$, lo cual puede justificar los hallazgos controversiales antes descritos.

En síntesis, si bien las variantes c.C677T y c.A1298C del gen MTHFR representan factores de riesgo para $\mathrm{MM}$ en otras poblaciones, su efecto podría haber ser modificado en Chile por la fortificación con ácido fólico más la influencia de otros factores no relacionados con el metabolismo de folatos que podrían ser los responsables de los casos de EB post fortificación. En esta serie de factores que explican la etiología del fenotipo en estudio, también pueden tener influencia el efecto del origen étnico de nuestra población, o bien las diferentes posiciones anatómicas de la EB que al parecer también tendrían diferentes orígenes etiológicos.

\section{Referencias}

1. Botto LD, Moore CA, Khoury MJ, Erickson JD. Neuraltube defects. N Engl J Med 1999; 341: 1509-19.

2. López-Camelo JS, Castilla EE, Orioli IM; INAGEMP (Instituto Nacional de Genética Médica Populacional); ECLAMC (Estudio Colaborativo Latino Americano de Malformaciones Congénitas). Folic acid flour fortification: impact on the frequencies of 52 congenital anomaly types in three South American countries. Am J Med Genet A 2010; 152A: 2444-58.

3. Cortés F, Mellado C, Pardo RA, Villarroel LA, Hertrampf E. Wheat flour fortification with folic acid: Changes in neural tube defects rates in Chile. Am J Med Genet A 2012; 158A:1885-90.

4. Green R. Is it time for vitamin B-12 fortification? What are the questions? Am J Clin Nutr 2009; 89: 712S-6S.

5. Rosenquist TH, Schneider AM, Monogham DT. $\mathrm{N}$ - methyl-D-Aspartate receptor agonists modulate homocysteine-induced developmental abnormalities. FASEB J 1999; 13: 1523-31.

6. Goyette P, Sumner JS, Milos R, Duncan AM, Rosenblatt DS, Matthews RG, et al. Human methylenetetrahydrofolate reductase: isolation of cDNA mapping and mutation identification. Nat Genet 1994; 7: 551.

7. Volcik KA, Blanton SH, Tyerman GH, Jong ST, Rott EJ, Page TZ, et al. Methylenetetrahydrofolate reductase and spina bifida: evaluation of level of defect and maternal genotypic risk in Hispanics. Am J Med Genet 2000; 95 : 21-7.

8. Van der Put NM, Eskes TK, Blom HJ. Is the common $677 \mathrm{C}-->\mathrm{T}$ mutation in the methylenetetrahydrofolate reductase gene a risk factor for neural tube defects? A meta-analysis. QJM 1997; 90: 111-5.

9. Amorim MR, Lima MA, Castilla EE, Orioli IM. NonLatin European descent could be a requirement for association of NTDs and MTHFR variant $677 \mathrm{C}>\mathrm{T}$ : a meta-analysis. Am J Med Genet A 2007; 143A: 1726-32.

10. Wang XW, Luo YL, Wang W, Zhang Y, Chen Q, Cheng YL. Association between MTHFR A1298C polymorphism and neural tube defect susceptibility: a metaanalysis. Am J Obstet Gynecol 2012; 206: 251.e1-e7.

11. Reyes-Engel A, Muñoz E, Gaitan MJ, Fabre E, Gallo M, Dieguez JL, et al. Implications on human fertility of the $677 \mathrm{C}-->\mathrm{T}$ and $1298 \mathrm{~A}-->\mathrm{C}$ polymorphisms of the MTHFR gene: consequences of a possible genetic selec- 
tion. Mol Hum Reprod 2002; 8: 952-7.

12. Mayor-Olea A, Callejón G, Palomares AR, Jiménez AJ, Gaitán MJ, Rodríguez A, et al. Human genetic selection on the MTHFR $677 \mathrm{C}>\mathrm{T}$ polymorphism. BMC Med Genet 2008; 9: 104

13. Nitsche F, Alliende MA, Santos JL, Pérez F, Santa María L, Hertrampf E, et al. [Frequency of C677T polymorphism of 5, 10-methylenetetrahydrofolate reductase (MTHFR) in Chilean mothers of spina bifida cases and controls]. Rev Med Chile 2003; 131: 1399-404.

14. Chomczynski P, Sacchi N. Single-step method of RNA isolation by acid guanidinium thiocyanate-phenolchloroform extraction. Anal Biochem 1987; 162: 156-9.

15. Spielman RS, McGinnis RE, Ewens WJ. Transmission test for linkage disequilibrium: the insulin gene region and insulin-dependent diabetes mellitus (IDDM). Am J Hum Genet 1993; 52: 506-16.

16. Dudbridge F. Likelihood-based association analysis for nuclear families and unrelated subjects with missing genotype data. Hum Hered 2008; 66: 87-98.

17. Cordell HJ, Barratt BJ, Clayton DG. Case/pseudocontrol analysis in genetic association studies: A unified framework for detection of genotype and haplotype associations, gene-gene and gene-environment interactions, and parent-of-origin effects. Genet Epidemiol 2004; 26: 167-85.

18. Guilmatre A, Sharp AJ. Parent of origin effects. Clin Genet 2012; 81: 201-9.

19. Weinberg CR. Methods for detection of parent-of-origin effects in genetic studies of case-parents triads. Am J Hum Genet 1999; 65: 229-35.

20. Hager R, Cheverud JM, Wolf JB. Maternal effects as the cause of parent-of-origin effects that mimic genomic imprinting. Genetics 2008; 178:1755-62.

21. Wilkins JF, Haig D. What good is genomic imprinting: the function of parent-specific gene expression. Nat Rev Genet 2003; 4: 359-68.

22. Dean JC, Moore SJ, Osborne A, Howe J, Turnpenny PD. Fetal anticonvulsant syndrome and mutation in the maternal MTHFR gene. Clin Genet 1999; 56: 216-20.

23. Bland JM, Altman DG. Statistics notes. The odds ratio. BMJ 2000; 320 (7247): 1468.
24. Rothhammer F, Lasserre E, Blanco R, Covarrubias E, Dixon M. Microevolution in human Chilean populations. IV. Shovel shape, mesial-palatal version and other dental traits in Pewenche Indians. Z Morphol Anthropol 1968; 60: 162-9.

25. Valenzuela CY, Harb Z. Socioeconomic assortative mating in Santiago, Chile: a demonstration using stochastic matrices of mother-child relationships applied to $\mathrm{ABO}$ blood groups. Soc Biol 1977; 24: 225-33.

26. Cruz-Coke R, Moreno RS. Genetic epidemiology of single gene defects in Chile. J Med Genet 1994; 31: 702-6.

27. Johnson VJ, Yucesoy B, Luster MI. Genotyping of single nucleotide polymorphisms in cytokine genes using realtime PCR allelic discrimination technology. Cytokine 2004; 27: 135-41.

28. Osaki R, Imaeda $\mathrm{H}$, Ban $\mathrm{H}$, Aomatsu $\mathrm{T}$, Bamba $\mathrm{S}$, Tsujikawa $\mathrm{T}$, et al. Accuracy of genotyping using the TaqMan PCR assay for single nucleotide polymorphisms responsible for thiopurine sensitivity in Japanese patients with inflammatory bowel disease. Exp Ther Med 2011; 2: 783-6.

29. López-Camelo JS, Orioli IM, da Graça Dutra M, NazerHerrera J, Rivera N, Ojeda ME, et al. Reduction of birth prevalence rates of neural tube defects after folic acid fortification in Chile. Am J Med Genet A 2005; 135: 120 5 .

30. Suazo J, Pardo R, Castillo S, Martin LM, Rojas F, Santos JL, et al. Family-Based Association Study Between SLC2A1, HK1 and LEPR Polymorphisms with Myelomeningocele in Chile. 2013. Reprod Sci 2013; 20: 1207-14.

31. Etheredge AJ, Finnell RH, Carmichael SL, Lammer EJ, Zhu H, Mitchell LE, et al. Maternal and infant genefolate interactions and the risk of neural tube defects. Am J Med Genet A 2012; 158A (10): 2439-46.

32. Pangilinan F, Molloy AM, Mills JL, Troendle J, ParleMcDermott A, Signore C, et al. Evaluation of common genetic variants in 82 candidate genes as risk factors for neural tube defects. BMC Medical Genetics 2012; 13: 62 .

33. Van Allen MI, Kalousek DK, Chernoff GF, Juriloff D, Harris M, Mcgillivray BC, et al. Evidence for multi-site closure of the neural tube in humans. Am J Med Genet 1993; 47: 723-43. 